\title{
Star cluster winds: The impact of radiative cooling
}

\author{
Sergiy Silich ${ }^{1}$, G. Tenorio-Tagle ${ }^{1}$ \\ and A. Rodríguez-González ${ }^{1}$ \\ ${ }^{1}$ Instituto Nacional de Astrofísica Optica y Electrónica, AP 51, 72000 Puebla, Mexico \\ email: silich@inaoep.mx
}

\begin{abstract}
Here we present a self-consistent stationary solution for spherically symmetric winds driven by massive star clusters under the impact of radiative cooling. We demonstrate that cooling may modify drastically the distribution of temperature and the observational appearance of star cluster winds if the rate of injected energy approaches a critical value, and that the stationary wind solution does not exist if the energy deposition rate exceeds this limit.
\end{abstract}

\section{The stationary wind solution}

The prototype model for adiabatic star cluster winds was proposed by Chevalier and Clegg (1985). In this presentation we re-examine this model taking into consideration the impact of radiative cooling.

In the adiabatic case there is an analytic solution which is completely defined by the star cluster radius $\left(R_{s c}\right)$ and the energy $\left(\dot{E}_{s c}\right)$ and mass $\left(\dot{M}_{s c}\right)$ deposition rates. In the radiative case one has to integrate the basic equations numerically that rises the problem of the initial conditions. To overcome this problem one has to realize that the type of the integral curve is dependent on the position of the sonic point with respect to the star cluster surface. The stationary wind solution results only if the sonic point lies at the star cluster surface. In turn, the position of the sonic point $R_{\text {sonic }}$ is defined by the central wind pressure $P_{c}$. The appropriate value of $P_{c}$ can be found by iteration until the sonic point takes its proper position at the star cluster surface.

\section{The radial wind structure and the threshold energy}

In the adiabatic case one can always find the value of $P_{c}$ that accommodates the sonic point at the star cluster surface. In the radiative solution $P_{c}$ cannot exceed a maximum value bound by the gas radiative cooling. At this critical stage there is no density enhancement able to compensate the fall in pressure promoted by radiative cooling and $R_{\text {sonic }}$ cannot be arbitrarily large. Figure 1 (left panel) displays the critical energy deposition rate for different values of $q_{e} / q_{m}$, where $q_{e}$ and $q_{m}$ are the energy and mass deposition rates per unit volume, respectively. Moving along the vertical line is equivalent to considering progressively more energetic star clusters, all with the same radius $R_{s c}$. When the energy deposition rate exceeds the critical value, the sonic point moves inside the star cluster and the stationary wind solution vanishes.

Star cluster winds present a four zone structure (Silich et al. 2003): a star cluster region filled with a hot X-ray plasma, an adjacent X-ray halo, the line cooling zone and a low temperature photoionized envelope. Figure 1 also shows the free wind temperature distribution for three SSCs, all with the same radius, but different energy deposition rates. The lowest energy case (solid line) lies well into the adiabatic regime. In the other 

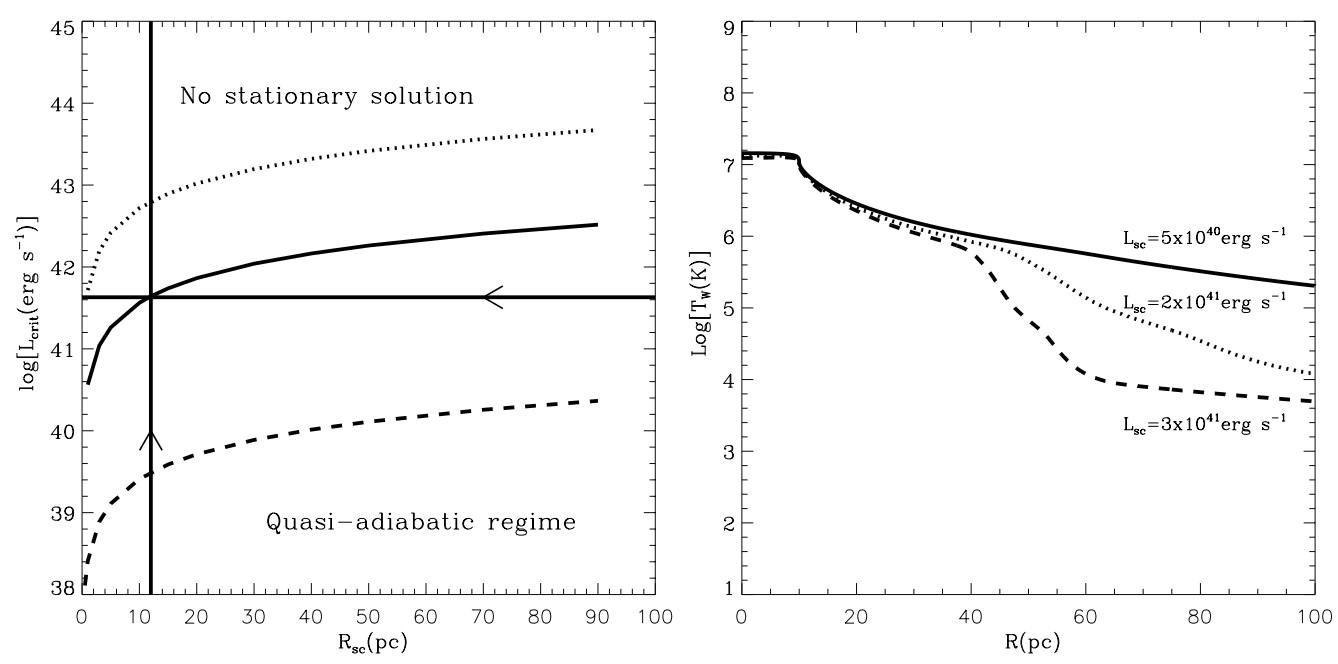

Figure 1. The impact of radiative cooling. The threshold energy input rate (left). The solid line represents the threshold energy for star clusters with $\left(2 q_{e} / q_{m}\right)^{1 / 2}=1000 \mathrm{~km} \mathrm{~s}^{-1}$. The dotted and the dashed lines mark the threshold energies for star clusters with $\left(2 q_{e} / q_{m}\right)^{1 / 2}=1500 \mathrm{~km}$ $\mathrm{s}^{-1}$ and $500 \mathrm{~km} \mathrm{~s}^{-1}$, respectively. Temperature profiles for progressively larger energy deposition rates (right). Solid, dotted and dashed lines represent winds with energy deposition rates $0.5,2$ and $3 \times 10^{41} \mathrm{erg} \mathrm{s}^{-1}$, respectively.

two cases, however, the radiative cooling clearly modifies the internal wind structure, increasing the maximum density and emission measure of the line cooling zone and photoionized envelope. This may be observed as a broad $\left(\sim 1000 \mathrm{~km} \mathrm{~s}^{-1}\right)$ emission line component of low intensity at the base of the narrow line caused by the central HII region.

\section{Conclusions}

A new self-consistent stationary solution for spherically symmetric radiative winds driven by compact star clusters is proposed. Our model predicts that the radial wind structure may be radically different from what is expected from the adiabatic solution. This implies a much less extended region of X-ray emission, a much denser photo-ionized gaseous envelope and a low intensity line emission coming from the compact line cooling zone and photo-ionized envelope.

For super-star clusters with super-critical energy input rates radiative cooling leads to a catastrophic cooling regime where the stationary wind solution is inhibited.

\section{Acknowledgements}

This study was supported by the México (CONACYT) research grant 36132-E.

\section{References}

Chevalier, R. A., \& Clegg, A. W. 1985, Nature, 317, 44

Silich, S., Tenorio-Tagle G., \& Muñoz-Tuñón, C. 2003, ApJ, 590, 796 